Voix et Images

\title{
Quand écrire c'était agir : la série culturelle québécoise au XIXe siècle
}

\section{Louis Francoeur}

Volume 6, numéro 3, printemps 1981

Philippe Haeck

URI : https://id.erudit.org/iderudit/200285ar

DOI : https://doi.org/10.7202/200285ar

Aller au sommaire du numéro

Éditeur(s)

Les Presses de l'Université du Québec

ISSN

0318-9201 (imprimé)

1705-933X (numérique)

Découvrir la revue

Citer cet article

Francoeur, L. (1981). Quand écrire c'était agir : la série culturelle québécoise au XIXe siècle. Voix et Images, 6(3), 453-463. https://doi.org/10.7202/200285ar d'utilisation que vous pouvez consulter en ligne.

https://apropos.erudit.org/fr/usagers/politique-dutilisation/ 


\title{
Quand écrire c'était agir: la série culturelle québécoise au XIXe siècle.
}

\author{
par Louis Francoeur
}

La critique littéraire au Québec accorde généralement peu d'importance à la littérature autochtone du XIXe siècle ${ }^{1}$. Envisagée du point de vue culturel. on déplore qu'elle ne soit pas de la même qualité esthétique que son aînée. la littérature française romantique; on évite de la comparer aux littératures anglo-saxonnes du Canada et des États-Unis sous prétexte qu'elle n'est pas une littérature comme les autres, qu'elle est même beaucoup moins une littérature que ne le sont plusieurs littératures nationales. En dernière analyse, on met en cause son existence même ${ }^{2}$.

Cette conception de l'ceuvre littéraire et de la série culturelle ${ }^{3}$ dans laquelle elle s'insère n'est pas sans trahir un certain nombre d'erreurs méthodologiques qui ont suffisamment eu cours dans le passé pour qu'on tente aujourd'hui de les identifier. En effet, les jugements plus ou moins sévères que la critique littéraire porte sur les œuvres du XIXe siècle québécois reposent sur de faux postulats que l'on peut résumer ainsi : refus de considérer l'œuvre littéraire comme signe autonome, persistance à juger de la valeur d'une œuvre d'une série donnée en utilisant des critères propres à une autre série, affirmation d'une croyance naïve au caractère arbitraire du lien entre l'expression et le contenu de l'œuvre tout aussi bien qu'entre l'œuvre et sa fonction, ignorance, feinte ou réelle, de la multifonctionnalité de l'œuvre et de la hiérarchie de ses fonctions, séparation radicale de la notion de valeur et des notions de structure et de fonction qui sont en réalité interdépendantes, omission surtout de prendre en compte la définition de la littérature que I'Institution de la série culturelle proposait. Or celle-ci, par la voix des premiers membres de l'Institut canadien au milieu du siècle dernier, considérait la culture des lettres et la civilisation comme une seule et même chose ${ }^{4}$, à telle enseigne que pour elle la littérature d'un peuple, c'est son histoire; c'est l'ensemble des écrits de ses citoyens les plus distingués, philosophes, savants, poètes, romanciers, jurisconsultes, politiques, prédicateurs, journalistes, en un mot, elle renferme à peu près toutes choses ${ }^{5}$. D'autres précisions seront apportées quelques années plus tard par la Société historique de Montréal qui refusera à son tour de fixer les limites de la littérature aux seules œuvres d'agrément et d'imagination. La littérature sera alors considérée comme l'expression par la parole écrite ou parlée du Vrai, du Bien et du Beau dans les idées et dans 
les sentiments et c'est à ce titre que la Philosophie, l'Histoire, le Droit, l'Économie sociale, l'Esthétique et l'Apologétique chrétienne en feront partie ${ }^{6}$. D'entrée de jeu il faut convenir qu'il en va autrement que d'une simple définition de la littérature. C'est en réalité tout un PROGRAMME unificateur des différents systèmes signifiants de la culture québécoise au XIXe siècle qui est proposé par l'Institution. A travers ses préoccupations morales, religieuses et patriotiques, celle-ci affirme l'unité nécessaire de l'activité culturelle de l'homme de ce temps et, par voie de conséquence, l'unité des systèmes signifiants qui en composent l'armature. Bien davantage, cette Institution refuse de reconnaître comme littéraire tout système signifiant qui prétendrait se développer de façon isolée. Comme cela se produit souvent dans I'histoire récente de la sémiotique, la recherche théorique a été précédée par des activités empiriques qui lui fournissent un véritable fondement, ce qui est plus qu'une pure présupposition comme l'affirment certains sémioticiens ${ }^{7}$. De son côté, la critique n'ayant pas vu que la structure de l'cuvre, sa valeur et sa fonction sont indissociablement liées au caractère unitaire de la série culturelle dans laquelle elle s'inscrit, se condamnait à chercher en dehors de sa série les critères qui doivent présider à la description et à l'analyse du texte-occurrence.

\section{Un champ théorique}

Pour les fins de notre étude, nous considérerons les seules œuvres de fiction romanesques, dramatiques et poétiques. Le concept de systèmes que nous venons d'évoquer à l'instant n'existe pratiquement et théoriquement que dans une relation de solidarité avec celui de modèle, défini comme système $S^{\prime}$ représentatif d'un système $S$ d'un degré inférieur d'abstraction. En conséquence, dans la première phase de la modélisation, la phase déductive, l'exploration d'un champ théorique doit permettre de trouver les critères d'identification des divers systèmes signifiants de la série culturelle en tant qu'unités fondamentales. A cet égard, nous proposons dans la démarche de validité de notre modèle une double articulation hiérarchisée avec croissance successive du degré d'abstraction. A un premier niveau, nous retiendrons que l'unité fondamentale, en tant que système modélisant secondaire, tout en participant du modèle cognitif dans la transmission d'une certaine vision du monde s'apparente aussi au modèle ludique par son aspect conventionnel et par les règles qui en régissent l'emploi. A un second niveau, nous prendrons en compte que les travaux sur les actes de langage de l'École analytique anglaise, particulièrement ceux de Austin et de Searle ${ }^{8}$, ont déjà permis au plan linguistique de reconnaître que l'unité minimale de communication n'est pas le symbole, le mot ou la phrase mais la PRODUCTION du symbole, du mot ou de la phrase au moment où se réalise l'acte de langage. Nous proposons de considérer l'ensemble $X$ d'éléments de base, les actes de langage de la communication linguistique, et l'ensemble $Y$ de règles d'organisation de ces éléments de base telles qu'énoncées par exemple par Searle pour l'acte de langage de demande, de promesse ou d'avertissement comme la formalisation d'un modèle théorique $\mathrm{M}^{\prime}$ dont le champ de validité s'étend au seul domaine de la pragmatique linguistique. De ce modèle théorique $M^{\prime}$ nous 
ferons découler un deuxième modèle $M$ " qui lui est homomorphe. En fait, nous posons une correspondance surjective entre les deux modes de caractérisation 9 . Ainsi, la production d'une occurrence de discours dans certaines conditions et suivant certaines règles sera tenue pour un acte de langage et cet acte de langage ou acte illocutionnaire de la littérature sera considéré comme une unité minimale de base de la communication culturelle. En vertu de la déontologie de l'Institution culturelle et des conventions littéraires par lesquelles le locuteur-auteur fait connaître ses intentions, la force illocutionnaire indiquera la signification particulière et complète à accorder à l'acte illocutionnaire littéraire et en dernière analyse déterminera également sa fonction particulière et complète dans la série culturelle. Selon cette hypothèse, l'activité créatrice de chaque locuteur-auteur aura consisté à adopter une forme de comportement soumis à des conditions et régi par des règles de telle sorte que «écrire, c'est agir», comme le suggère l'abbé Casgrain à propos du Jean Rivard, le défricheur d'Antoine Gérin-Lajoie dont il dit que c'est plus qu'un bon livre, c'est une bonne ACTION 10 .

La phase prévisionnelle, la seconde phase de la modélisation, consistera pour nous à poser la prédiction que grâce à ce modèle théorique, le test subi par chaque discours de fiction de la série culturelle québécoise du XIXe siècle en tant qu'acte de langage d'exhortation patriotique sera positif. Conséquemment, nous supposerons que, possédant la même force illocutionnaire, ils auront la même valeur et rempliront la même fonction et qu'en dernière analyse ils seront les constituants fondamentaux de la série culturelle.

Nous parcourrons la phase descriptive aux trois niveaux de l'acte illocutionnaire littéraire.

\section{Les actes d'énonciation}

En premier lieu, il apparaît avec évidence qu'à l'intérieur de cette même série culturelle, les ACTES D'ÉNONCIATION sont protéiformes et obéissent à des lois génériques de structuration diverses. Ainsi apparaissent simultanément au Québec dans le milieu du XIXe siècle des discours romanesques, poétiques et dramatiques qui n'ont de commun au plan de l'acte d'énonciation que d'utiliser une suite de mots, de phrases ou de dialogues en conformité avec les lois de leur genre respectif. Cependant on devrait éviter de croire, comme le font trop facilement certains critiques, que ces actes d'énonciation différents produits par des locuteurs différents sont tout naturellement porteurs de contenus différents. Au contraire, dans cette même série culturelle, tout en effectuant des actes d'énonciation hétérogènes génériquement, les locuteurs semblent avoir accompli des ACTES PROPOSITIONNELS et ILLOCUTIONNAIRES homogènes. A certaines conditions et suivant certaines règles toutefois.

\section{Les actes de contenu propositionnel}

Tous les actes illocutionnaires de la littérature d'une même série culturelle contenant la même référence et la même prédication et la signi- 
fication des expressions référentielles étant aussi la même, nous pouvons conclure que l'acte de contenu propositionnel est identique dans tous les cas. Ce contenu $(r / p)$ a pu être identifié grâce aux différents marqueurs externes et internes qui précèdent ou parsèment les actes illocutionnaires littéraires. Les titres ou sous-titres constituent en effet des marqueurs externes de contenu propositionnel de première importance. Que ce soit Jacques Cartier ou Canada vengé ou bien Jacques et Marie, souvenir d'un peuple dispersé ou Le Drapeau de Carillon, ces titres ou sous-titres désignent les expressions référentielles de l'Histoire ou les expressions prédicatives de l'agir patriotique ou parfois même les deux réunies comme dans la proposition-titre Les exploits d'lberville. Les préfaces renferment d'autres marqueurs externes qui peuvent rendre explicite le contenu propositionnel de l'acte illocutionnaire, comme l'illustrent ces lignes: "Chaque page de notre histoire renferme un drame. Eh bien! dramatisons l'Histoire du Canada, faisons mouvoir devant les yeux de notre peuple ces grandes figures de nos annales avec leurs vertus, leurs passions, et le peuple nous lira $11 \%$. A ces marqueurs externes de contenu propositionnel sont venus s'ajouter des marqueurs internes que sont les catégories sémantiques récurrentes ou isotopies, celle de l'Histoire et celle de l'agir patriotique.

L'expression référentielle définie comme l'expression utilisée par un locuteur pour poser une référence trouvera son analogon dans le personnageacteur, lieu privilégié de l'expression du contenu de l'énoncé. L'emploi du personnage-référentiel est soumis à l'axiome d'existence ainsi formulé : «Tout ce à quoi on réfère doit exister ${ }^{12}$ \%. Ce principe d'existence s'applique doublement dans le cas présent puisque le personnage-référentiel est utilisé pour référer à l'univers de l'œuvre tout aussi bien qu'à l'univers historique réel. A titre d'exemple, nous soulignerons que les nombreux personnages Jacques Cartier, tout en étant utilisés pour référer au découvreur du Canada, sont également des personnages-acteurs qui réfèrent à l'univers de l'œuvre non pas comme des copies conformes mais bien plutôt comme des unités de sens. Le principe d'existence préside ainsi à l'élaboration et à la conservation de la référence comme acte de langage en tant que signifié autonome et communicable.

Cette communication a lieu en conformité avec un deuxième principe dit d'identification selon lequel «Toute expression référentielle a forcément un sens ${ }^{13}$ \%. Le personnage-référentiel, en effet, ne réfère pas par lui-même mais permet plutôt au locuteur de communiquer à l'allocutaire (lecteur ou spectateur) des faits uniques, dont ceux qui constituent le passé historique des Québécois. En conformité avec les règles, les personnages-référentiels acquièrent leur sens, leur signification, leur valeur même. Leur sens est leur rôle cardinal dans l'économie de l'acte illocutionnaire littéraire. Antérieur à la référence, le sens que portent les personnages-référentiels doit permettre à l'allocutaire d'identifier le référent ou au besoin d'en fournir une description identifiante qui autorise à juger s'il a saiși ce qui est signifié dans l'acte de référence. Ceci ne sera possible que si, préalablement, l'expression référentielle a été utilisée en conformité avec les règles qui la gouvernent et que nous pouvons énoncer ainsi: 
Regle 1: le personnage-référentiel (r), disons Papineau, n'apparaît que dans des actes d'énonciation, disons telle pièce de théâtre, susceptibles de constituer l'accomplissement d'un acte illocutionnaire littéraire.

Règle 2: ( $\mathrm{r}$ ) n'est employé que s'il existe un personnage historique $(\mathrm{H})$ tel que ou bien ( $r$ ) contienne une description identifiante de $(H)$ ou bien $(L)$ (le locuteur-auteur) soit capable de compléter (r) par une description de $(H)$ ou de son contexte et tel que, par l'emploi de (r), (L) ait l'intention d'identifier $(\mathrm{H})$ ou son contexte pour (A) (l'allocutaire lecteur ou spectateur).

Règle 3: Utiliser $(r)$ revient à identifier $(\mathrm{H})$ à l'intention de (A).

L'utilisation que nous avons faite de ces règles ${ }^{14}$ dans la série culturelle étudiée nous permet de déduire deux choses: premièrement, les actes de langage de la littérature au Québec au XIXe siècle possèdent des personnagesréférentiels distincts mais dont le sens et la référence sont identiques en vertu des règles qui en gouvernent l'usage unique; deuxièmement, leurs locuteurs posent la même référence parce que animés de la même intention.

Si nous avons pu attribuer aux personnages-acteurs le rôle de l'expression référentielle, nous réserverons l'expression prédicative aux actes de description ou de caractérisation de ces mêmes personnages à l'intérieur de l'acte illocutionnaire littéraire. Ainsi, la prédication qui marque le XIXe siècle québécois tient-elle complètement et uniquement dans l'attribution de l'agir patriotique aux personnages-référentiels, de telle sorte que la proposition unique et intégrale de tous les actes illocutionnaires, $(r / p)$, serait la suivante: «le personnage historique de fiction $(r) /$ agir patriotique $(p)$ ». Cependant $(r / p)$ se retrouvant toujours à l'intérieur d'un acte illocutionnaire, la formule doit devenir pour être complète: $f(r / p)$, dans laquelle la variable "f» obtient ses différentes valeurs des diverses forces illocutionnaires qu'elle représente. Ces modifications de valeurs affectent directement l'expression prédicative. En l'occurrence, les procédés marqueurs de force illocutionnaire vont déterminer le mode dans lequel l'agir patriotique est prédiqué à propos du personnage historique de fiction. Or tous les actes illocutionnaires littéraires étudiés dans cette série, y compris ceux qui relèvent du genre poétique, sont régis par les mêmes procédés marqueurs de force illocutionnaire, ceux de l'assertion narrative. "f» (je raconte que) agit sur le prédicat (agir patriotique) de telle façon qu'il assigne le mode dans lequel le prédicat se rapporte à l'objet (personnage historique) auquel réfère le terme référentiel (personnage de fiction) ${ }^{15}$. Ceci permet d'expliquer que des personnages historiques tenus pour suspects quant à leurs intentions véritables et à leurs actions réelles comme l'affirment certains historiens au sujet de Dollard des Ormeaux, soient devenus des modèles de vertu patriotique dont les locuteurs-auteurs racontent les exploits en assurant leurs allocutaires que $(r / p)$ est vrai. Le fondement de cette démarche pragmatique réside dans le fait que le prédicat (agir patriotique) a pour rôle de préciser à quoi s'engage le locuteur lui-même en rapport avec l'Histoire. Il le fait en se conformant à certaines règles que nous pouvons énoncer ainsi: 
Règle 1: p (agir patriotique) n'a été énoncé que dans le contexte d'un discours T dont l'énoncé peut constituer l'accomplissement d'un acte illocutionnaire littéraire.

Règle 2: p (agir patriotique) n'est énoncé dans le discours $\mathrm{T}$ que si l'énoncé de $\mathrm{T}$ implique une référence effective à $\mathrm{H}$ (personnage historique).

Regle 3: p n'est énoncé que si $H$ appartient à une catégorie qu'il est logiquement possible que $p$ soit vrai ou faux de $\mathrm{H}$.

Règle 4: l'énoncé de $\mathrm{p}$ revient à soulever la question de la vérité ou de la fausseté de $\mathrm{p}$ à propos de $\mathrm{H}^{16}$.

\section{Les intentions}

L'expression de la proposition $(r / p)$ a nécessairement lieu à l'intérieur d'un acte illocutionnaire 17 littéraire dont nous avons déjà dit qu'il prenait ici la forme de $f(r / p)$ dans laquelle la variable « $f$ » était marqueur de force illocutionnaire de narrativité assertive de telle sorte que tous les discours de fiction pourraient être transcodés selon la proposition linguistique suivante: « je raconte que les héros historiques agissent de façon patriotique». Cependant, il faut également prendre en compte la recherche d'une autre signification que voulait produire chaque locuteur dans l'accomplissement de son acte illocutionnaire littéraire et qui vient s'ajouter à celle de raconter. Les préfaces des romans, par exemple, illustrent à l'envi cette nécessité qu'éprouvent les locuteurs de voir cette autre intention reconnue par leurs allocutaires, comme en témoignent ces lignes qui peuvent résumer tous ces propos liminaires : «Ces pages que j'ai consacrées à (la) mémoire (de nos pères) et que je vous offre (...). si elles peuvent faire verser quelques larmes nouvelles sur les souffrances oubliées (...) si elles servent à retremper nos cœurs dans leur foi et leurs vertus de toutes sortes, et nous engagent à imiter leur exemple dans toutes les circonstances difficiles qui sont encore réservées à notre existence nationale, alors je n'aurai pas entrepris une tâche inconsidérée ${ }^{18_{x}}$.

\section{Les conventions}

La signification que les locuteurs voulaient transmettre par la reconnaissance de cette intention ne pouvait être atteinte que par l'utilisation d'un certain nombre de procédés culturels conventionnels. Ces conventions qui étaient assurément nécessaires à l'élaboration et à la production de l'acte de langage, l'étaient tout autant pour l'allocutaire dans son entreprise de décodage. Elles découlent, en effet, logiquement d'un système de règles établi, reconnu ou entériné par l'Institution de la série culturelle. Quand, par exemple, l'abbé Casgrain, chef de file du mouvement littéraire de Québec, écrivait: «Ainsi sa voie (celle de la littérature) est tracée d'avance: elle sera le miroir fidèle de notre petit peuple, dans les diverses phases de son existence, avec sa foi ardente, ses nobles aspirations, ses élans d'enthousiasme, ses traits d'héroïsme, sa généreuse passion de dévouement. Elle n'aura point ce cachet de réalisme moderne, manifestation de la pensée impie, matérialiste; mais elle 
n'aura que plus de vie, de spontanéité, d'originalité d'action $19^{\text {», il énonçait les }}$ règles que l'Institution littéraire souhaitait voir suivre par les locuteurs-auteurs. Et nombreux sont ceux qui ont clairement manifesté leur intention de s'y conformer docilement. Comment pouvait-il en être autrement? Écrire étant une forme de comportement régi par des règles, il devenait impérieux pour eux que ces mêmes règles soient respectées afin que la signification de leur acte de langage littéraire soit reconnue. Règles constitutives qui *créent et définissent de nouvelles formes de conduite ${ }^{20}$, que nous pouvons regrouper sous l'appellation de conduite patriotique, règles normatives qui gouvernent des formes de conduite déjà existantes et qui ont trait notamment à une certaine conception et une certaine utilisation des genres littéraires en vigueur dans la série culturelle, comme par exemple le modèle qu'offrait pour le roman historique l'œuvre de Walter Scott. Ainsi le genre romanesque deviendra-t-il "récit historique édifiant» et le genre dramatique «drame historique», se conformant en cela au rejet par l'Institution des œuvres de fiction romanesques et dramatiques toutes aussi «frivoles et scandaleuses" qui avaient cours dans la série culturelle française de la même époque.

\section{La sanction}

La nécessité qu'éprouvent les locuteurs de se conformer aux règles et conventions de l'Institution, de respecter dans l'écriture le caractère de légisigne de l'acte illocutionnaire littéraire, se manifeste avec une grande régularité dans la recherche explicite de la sanction positive de l'allocutaire. Une sanction négative signifierait en effet le rejet de l'ceuvre de la série littéraire. On a ainsi assisté à la disparition de la revue La Ruche littéraire, frappée de la sanction sévère du critique de La Minerve pour avoir osé publier La vie d'un faux dévôt, roman qui avait échoué à «faire honneur à notre pays et à notre nationalité». D'où toute la gamme de propos liminaires qui vont de l'indifférence feinte du «lecteur jugera lui-même» à l'humble sollicitation de «A vous chers lecteurs de rendre complets ces vœux d'un grand cœur, en accueillant ce nouveau-né avec toute l'indulgence dont vous êtes capables ${ }^{21}$ ».

\section{La valeur illocutionnaire}

Si l'impérialisme des conventions se manifeste de façon aussi catégorique, si la sanction prend une telle importance pour les locuteursauteurs, nous pouvons à coup sûr y déceler l'affirmation du lien nécessaire qui les unit à la force illocutionnaire de l'énoncé. Pour accomplir l'acte illocutionnaire littéraire que l'on attend d'eux, les locuteurs doivent non seulement connaître les conventions qui régissent leur série culturelle mais ils doivent de plus se laisser gouverner par elles. Ces conditions étant satisfaites, la force illocutionnaire et son caractère conventionnel confèrent à l'œuvre la VALEUR que nous devons lui attribuer dans la série culturelle dans laquelle elle s'insère. A cet égard, la force illocutionnaire que nous retrouvons dans les œuvres littéraires québécoises du XIXe siècle découle logiquement des conventions qui ont déjà été identifiées. Les nombreux marqueurs externes et internes 
qui indiquent la façon dont if faut considérer le contenu propositionnel des différents discours sont sensiblement les mêmes. En effet, tout aussi bien par les titres et par les préfaces que par l'armature même de la syntagmatique narrative et discursive que les locuteurs n'hésitent pas à interrompre pour glisser un «Ami lecteur... qui les autorise à juger la conduite des personnages et à émettre des opinions personnelles sur tel ou tel problème historique, économique ou politique, la présence de ces marqueurs que Peirce appellerait des subindices indique justement qu'il faut lire et entendre ces discours comme autant d'exhortations. Exhorter la jeunesse à défricher la terre paternelle, à imiter les anciens Canadiens et leurs vertus, à admirer le courage et la persévérance de Jacques Cartier ou de d'lberville, telle est la valeur illocutionnaire que nous devons attribuer à toute la littérature historique de cette série culturelle. C'est dire du même coup que chaque acte de raconter est pris en charge par un acte illocutionnaire intégrant dont les limites de validité sont celles-là mêmes de la série culturelle qui lui impose son Institution, ses conventions et ses sanctions. Si sa force illocutionnaire (F) en est une d'exhortation, son référent (R) est le peuple tout entier, représenté dans ses allocutaires à qui on prédique l'imitation $(P)$ de ce qui est raconté $(f(r / p))$. La formule devient alors: $F((R / P) f(r / p))$, que nous pouvons traduire ainsi: "J'exhorte le lecteur à imiter ce que je raconte de l'agir patriotique des héros historiques». L'emploi des marqueurs de force illocutionnaire d'exhortation s'est exercé en conformité avec les règles qui le gouvernent et que nous pouvons énoncer en ces termes ${ }^{22}$ :

1. Règle de contenu propositionnel: Acte futur C (imitation par l'allocutaire A (le peuple).

\section{Règles préliminaires:}

2. 1: $L$ (locuteur) a des raisons de penser (en vertu de son appartenance à la série culturelle) que $C$ sera profitable à $A$.

2.2: Il n'est certain, ni pour $L$ ni pour $A$ que $A$ serait conduit à effectuer $C$ de toute façon (en vertu de la loi de Zipf, dite loi du moindre effort, ou du contexte social).

3. Règle de sincérité: $\mathrm{L}$ pense que $\mathrm{C}$ sera profitable à $\mathrm{A}$.

4. Règle essentielle: Elle revient à assumer que $\mathrm{C}$ sera profitable à $\mathrm{A}$.

Ces règles et tout particulièrement la règle de sincérité et la règle essentielle posent les actes illocutionnaires de la littérature en tant que porteurs d'une signification de vérité, comme nous l'avons déjà remarqué à propos de l'énoncé de l'expresssion prédicative qui soulève la question de vérité (règle 4) de $\mathrm{P}$ à propos de $\mathrm{H}$.

\section{L'effet perlocutionnaire}

Si l'acte illocutionnaire littéraire est par nature sincère et vrai, nous devons en déduire que pour l'allocutaire il existera toujours un point de vue 23 qui le conduira à considérer cet acte comme vrai. La conséquence de la véracité 
de ce dernier entraîne la considération des effets qu'il peut produire sur les pensées, les actions, les comportements des allocutaires. Par exemple, dans la série culturelle étudiée, la vérité de la prédication de l'agir patriotique au personnage de fiction et la sincérité des locuteurs dans l'énoncé de leur exhortation patriotique peuvent en effet convaincre ou inspirer les allocutaires, en d'autres mots rendre «les lectures utiles et instructives» pour le service de la patrie, comme le souhaitait un des porte-parales de l'Institution. Cette «bonne action» est un acte perlocutionnaire dont la nature a quelque affinité avec celle de la fonction sociale de l'ceuvre. De même que la fonction d'un système ne remplit pas toujours et obligatoirement son rôle social, de même l'acte illocutionnaire littéraire n'a pas toujours et obligatoirement d'effets perlocutionnaires. II a pu arriver effectivement que nos auteurs aient "prêché dans le désert». Mais de façon théorique, nous devons prendre en compte que les actes illocutionnaires peuvent être aussi des actes perlocutionnaires. Pratiquement, nous possédons de nombreux témoignages que des effets perlocutionnaires ont été produits dans notre série culturelle, témoignages que nous regrouperons sous les trois objectifs chers au romantisme nationaliste: CONVAINCRE de la mission providentielle de la nation, EDIFIER par l'exaltation de la race, ECLAIRER par le culte du héros. De telle façon qu'à l'acte illocutionnaire unique d'exhortation patriotique correspond dans la série culturelle un acte perlocutionnaire unique qui est celui de former la conscience nationale par la connaissance du passé. C'est affirmer du mêrme coup que la série culturelle pourra être décrite comme l'ensemble des actes illocutionnaires littéraires achevés dans les actes perlocutionnaires et leurs effets.

\section{La série culturelle}

L'unité des actes illocutionnaires littéraires au sein de la même série culturelle provient des relations fonctionnelles qu'ils entretiennent grâce à leur dimension perlocutionnaire. Mais unité n'est pas identité, comme convaincre n'est pas synonyme d'éclairer ou d'édifier, même si les trois actes peuvent également concourir à former la conscience nationale. Nous voulons suggérer par là qu'il existe des degrés de force perlocutionnaire comme il existe, suivant la suggestion de Chomsky, des degrés de grammaticalité25. Nous ouvrons ainsi la voie à une organisation hiérarchique uificatrice ${ }^{26}$ des actes illocutionnaires dans la série culturelle. La hiérarchie sera fondée, en effet, sur la présence dans la série culturelle d'un acte dominant dont le statut sera établi à partir de sa validité à procurer aux autres actes non seulement leur contenu propositionnel (leur structure sémantique) mais tout aussi bien leur force illocutionnaire (leur valeur) et leurs effets perlocutionnaires (donc leur fonction). L'Histoire du Canada de F.-X. Garneau s'est mérité ce privilège d'occuper le sommet dans la hiérarchie des actes illocutionnaires culturels au Québec au $\mathrm{XIXe} \mathrm{siècle} \mathrm{en} \mathrm{faisant} \mathrm{revivre} \mathrm{pour} \mathrm{la} \mathrm{première} \mathrm{fois} \mathrm{les} \mathrm{faits} \mathrm{passés} \mathrm{dans} \mathrm{le} \mathrm{but}$ d'exhorter la collectivité à prendre conscience de son présent pour assumer son avenir. «À la cause que nous avons embrassée dans ce livre, y lisons-nous, la conservation de notre religion, de notre langue et de nos lois, se rattache aujourd'hui notre destinée ${ }^{27}$. Tous les autres actes, roman historique, théâtre 
patriotique, poésie nationaliste, se sont organisés en hiérarchie avec croissance successive de leur vérité propositionnelle, de leur valeur illocutionnaire et de leur effet perlocutionnaire dans leur interrelation avec cet acte dominant. La durée du rôle structurant de ce dernier et sa zone de prégnance à l'endroit des autres actes ont permis de délimiter les frontières temporelles et spatiales de cette série culturelle.

Le type d'acte illocutionnaire dominant avec ses actes intégrés détermine en dernière analyse le caractère locutarisé ou allocutarisé de la série culturelle. Ainsi, une série culturelle comme celle que nous avons étudiée, largement dominée par l'acte de langage historique où les intentions des locuteurs et de l'Institution qui les gouverne font en sorte que le contenu propositionnel des actes illocutionnaires soit la vérité historique, la valeur illocutionnaire soit l'exhortation et la fonction perlocutionnaire soit la conviction en vue d'un combat à venir, cette série sera dite allocutarisée. Elle s'oppose en cela dans I'histoire culturelle du Québec à cette autre série plus récente que nous pouvons qualifier de locutarisée, âge de la parole poétique et du roman modélisé par l'autobiographie, dans laquelle l'acte illocutionnaire dominant est essentiellement poétique avec un contenu propositionnel sui-référentiel, une force illocutionnaire d'interrogation et une fonction perlocutionnaire de recherche d'identité.

La phase inductive de la modélisation devrait nous permettre de mesurer l'écart qui existe entre le modèle hypothétique des actes illocutionnaires littéraires et le modèle théorique des actes de langage "stricto sensu ". Cet écart, faut-il le souligner, participe des grandeurs et misères de la métaphore conçue comme instrument heuristique ${ }^{28}$, singulièrement lorsqu'elle est utilisée au second degré comme ce fut le cas dans cette étude. Si l'identité de structures entre le modèle théorique logico-linguistique $\mathrm{M}^{\prime}$ ' et le modèle théorique littéraire $M$ " permet de «parler d'une certaine façon 29 » qui est identique dans les deux cas, il n'en demeure pas moins que le problème de la référence métaphorique se trouve doublement posé par l'utilisation de $M^{\prime \prime}$. La mesure de l'écart entre l'objet à décrire et $M^{\prime \prime} d$ 'une part, et $M^{\prime \prime}$ et $M^{\prime} d^{\prime}$ 'autre part, semble à toutes fins utiles impossible. Faudra-t-il renoncer pour autant à recourir aux modèles logico-linguistiques pour fonder une démarche adéquate en sémiotique littéraire et culturelle? Le problème épistémologique que soulève cette interrogation nous semble déjà trouver quelques éléments de réponse dans le caractère heuristique de la fiction utilisée ici même pour proposer une nouvelle lecture d'une série culturelle importante du XIXe siècle québécois.

1. Cet article est une version remaniée d'une communication présentée au lle Congrès de l'Association internationale de sémiotique tenu à Vienne en juillet 1979.

2. J.-C. Falardeau, Notre société et son roman, Montréal, H.M.H., 1967, pp. 47-48.

3. Il ne faut pas confondre ici la notion de série culturelle avec les notions naïves d'époque ou de période qui reposent sur la seule unité de temps. Nous définissons une série culturelle comme un polysystème composé de plusieurs unités de signification (littérature, peintre, art et tradition populaire, etc.) qui sont elles-mêmes 
des sous-systèmes du premier et qui ont pour caractéristiques communes (1) d'être en interaction continue, (2) à l'intérieur d'une hiérarchie avec croissance successive, (3) dont le sommet est occupé par une œuvre ou un ensemble d'ceuvres qui agit comme principe premier structurant, (4) et dont la durée du rôle structurant et la sphère de prégnance permettent de délimiter les coordonnées spatiotemporelles.

4. Antoine Gérin-Lajoie, La Revue canadienne, no 4, 25 janvier 1845.

5. P.J.O. Chauveau, "Etat de la littérature en France depuis la Révolution", dans J. Huston, Le Répertoire national, 2, p. 209.

6. Napoléon Bourassa, La Revue canadienne, vol. 1, no 1, janvier 1864, p. 4.

7. V. V. Ivanov et al., "Thèses pour l'étude sémiotique des cultures", Recherches internationales à la lumière du marxisme, Paris, 1974.

8. J.L. Austin, Quand dire c'est faire, (How to do things with words, Oxford University Press, 1962), trad. fr. Gilles Lane, Paris, Seuil, 1970.

J.R. Searle, Les actes de langage, (Speech Acts, Cambridge University Press, 1969), trad. fr. Hélène Pauchard, Paris, Hermann, 1972.

9. Marie et Louis Francœur, «Deux contes nord-américains considérés comme actes de langage narratifs". Études litteraires, vol. 8, no 1, avril 1975, pp. 57-80, et Louis Francour, "Pour une typologie du monologue intérieur", Neohelicon, BudapestAmsterdam, IV, 3-4, 1976, pp. 137-166.

10. H.R. Casgrain dans I'Opinion publique du 26 février 1872, «Silhouettes littéraires», reproduit par Aug. Laperrière dans Les Guêpes candiennes, vol. 1, Ottawa, Bureau, 1881 , p. 226.

11. Edmond Rousseau, Préface à Les exploits d'lberville, Québec, C. Darveau, 1888.

12. Searle, op. cit., p. 121.

13. Ibid., p. 124.

14. Ibid., p. 142.

15. Ibid., p. 171.

16. Ibid., p. 176.

17. Ibid., p. 68.

18. Napoléon Bourassa, Jacques et Marie, Montréal, Eusèbe Sénécal, 1866.

19. H.R. Casgrain, «Le mouvement littéraire au Canada», Le Foyer canadien, IV, 1866.

20. Searle, op. cit., p. 72.

21. Edmond Rousseau, op. cit.

22. Searle, op. cit., p. 108.

23. Ju. M. Lotman et A.M. Pjatigorskij, «Le texte et la fonction", Semiotica, Mouton, La Haye, 1969, no 2, p. 208.

24. Searle, op. cit., p. 62.

25. Noam Chomsky, Structures syntaxiques, Paris, Seuil, 1969, p. 41.

26. Ivanov et al., "Ettude des cultures", Recherches internationales, Paris, 1974, p. 155

27. F.-X. Garneau, Histoire du Canada, Québec, 1859, t. I, 3e éd. revue et corrigée. Préface p. 8.

28. Paul Ricoeur, La métaphore vive, Paris, Seuil, 1975, p. 302.

29. Max Black، cité par Paul Ricoeur, La métaphore vive, p. 304. 